\title{
ESTRUCTURA LOCAL DE LOS PUNTOS DE TANGENCIA DEL TRANSFORMADO ESTRICTO DE UNA FOLIACIÓN POR CURVAS EN DIMENSIÓN 3
}

\section{Renato Benazic Tomé ${ }^{1}$}

RESUMEN.- En el presente trabajo, considerar campos vectoriales holomorfos de dimensión compleja 3 definidos en una vecindad del origen tales que $0 \in C^{3}$ es una singularidad aislada del tipo dicrítico y después de una explosión, su transformado estricto no presenta singularidades en el divisor. En estas condiciones, probaremos que en una vecindad de los puntos de tangencia, la foliación transformada estricta admite una forma normal.

\section{INTRODUCCIÓN}

Sea $U$ un subconjunto abierto de $\mathbb{C}^{n}(n \geq 2)$ tal que $0 \in \mathrm{U}$ y consideremos $Z=\sum_{i=1}^{n} Z_{i} \frac{\partial}{\partial z_{i}}$ un campo vectorial holomorfo definido en $U$ tal que $Z_{1}, Z_{2}, \ldots, Z_{n}$ no tengan divisores comunes. El Sistema Lineal de Ecuaciones Diferenciales Ordinarias Complejas asociado al campo $Z$ es dado por:

$$
\mid \begin{array}{ccc}
\frac{d z_{1}}{d T} & =Z_{1}\left(z_{1}, z_{2}, \ldots, z_{n}\right) \\
\frac{d z_{2}}{d T} & = & Z_{2}\left(z_{1}, z_{2}, \ldots, z_{n}\right) \\
\vdots & \vdots & \vdots \\
\frac{d z_{n}}{d T} & = & Z_{n}\left(z_{1}, z_{2}, \ldots, z_{n}\right)
\end{array}
$$

en donde $T \in \mathbb{C}$. Las soluciones de (1) definen una foliación singular por curvas complejas de $U$, la que denotaremos por $\mathcal{F}_{Z}$, cuyas singularidades son los ceros de $Z$.

Como cada $Z_{i}$ es una función de varias variables complejas definidas en una vecindad abierta del punto $p=\left(p_{1}, \ldots, p_{n}\right) \in \mathbb{C}^{n}$, entonces ella admite un desarrollo en serie de Taylor

\footnotetext{
${ }^{1}$ Universidad Nacional Mayor de San Marcos. Facultad de Ciencias Matemáticas. 


$$
Z_{i}=\sum_{k=0}^{\infty} Z_{i}^{k}
$$

en donde los $Z_{i}^{k}$ son polinomios homogéneos de grado $k$ en las variables complejas $z_{1}-p_{1}, \ldots, z_{n}-p_{n}$. El orden de $Z_{i}$ en el punto $p \in \mathbb{C}^{n}$, denotado por $\operatorname{ord}_{p}\left(Z_{i}\right)$ es, por definición, el menor número entero $v_{i}$ tal que $Z_{i}^{k} \equiv 0$ para todo $k<v_{i}$ y $Z_{i}^{v_{i}} \not \equiv 0$. La multiplicidad algebraica de la foliación $\mathcal{F}_{Z}$ (o del campo $Z$ ) en el punto $p$, denotada por $m_{p}(Z)$ es definida como el mínimo de los órdenes $\operatorname{ord}_{p}\left(Z_{i}\right)$. Decimos que, $p$ es un punto singular de $\mathcal{F}_{Z}$ (o de $Z$ ) si y sólo si $m_{p}(Z) \geq 1$, caso contrario $p$ es llamado punto regular. El conjunto de todos los puntos singulares de $\mathcal{F}_{Z}$ o $Z$ será denotado por $\operatorname{Sing}\left(\mathcal{F}_{z}\right)$ o simplemente por $\operatorname{Sing}(Z)$.

En el presente estudio, asumimos que $0 \in \mathbb{C}^{n}$ es él único punto singular de $Z$ en $U$. De esta manera la foliación $\mathcal{F}_{Z}$ es regular en $U$ excepto en cero.

Si $E: \tilde{U} \rightarrow U$ es el "blow-up" centrado en $0 \in \mathbb{C}^{n}$ (ver [2], [10], [12], [14]) entonces existe una única manera de extender el "pull-back" $E^{*}\left(\mathcal{F}_{z}-\{0\}\right)$ a una foliación singular analítica $\tilde{\mathcal{F}}_{z}$ sobre una vecindad del divisor excepcional $D=\mathbb{C} P(n-1)=E^{-1}(0) \subseteq \tilde{U}$ de tal manera que su conjunto singular sea de codimensión mayor que o igual a 2. En este caso, decimos que $\tilde{\mathcal{F}}_{z}$ es el transformado estricto de $\mathcal{F}_{Z}$ por el "blow-up" $E$. Decimos que, $0 \in \mathbb{C}^{n}$ es una singularidad no dicrítica de $\mathcal{F}_{Z}$ (o de $Z$ ) si y sólo si el divisor $D$ es invariante por $\tilde{\mathcal{F}}_{z}$, es decir $D$ es unión de hojas y singularidades de $\mathcal{F}_{Z}$. Caso contrario, $0 \in \mathbb{C}^{n}$ es llamado singularidad dicrítica.

Sea $m_{0}(Z)=v\left(v \in \mathbb{Z}^{+}\right)$, entonces las componentes $Z_{i}$ del campo $Z$ tienen desarrollo en serie de Taylor $Z_{i}=\sum_{k \geq v}^{\infty} Z_{i}^{k}(1 \leq i \leq n)$, en $0 \in \mathbb{C}^{n}$, donde cada $Z_{i}^{k}$ es un polinomio homogéneo de grado $k$. Si denotamos $Z^{k}=\sum_{i=1}^{n} Z_{i}^{k} \frac{\partial}{\partial z_{i}}$ (Observe que $Z^{k}$ es un campo polinomial homogéneo de grado $k \geq v$ ), entonces los siguientes enunciados son equivalentes (ver [1]):

1. $0 \in \mathbb{C}^{n}$ es una singularidad dicrítica de $Z$. 
2. $z_{j} Z_{i}^{v}-z_{i} Z_{j}^{v}=0, \forall 1 \leq i<j \leq n$.

3. $Z_{v}=P_{v-1} R$, donde $R=\sum_{i=1}^{n} z_{i} \frac{\partial}{\partial z_{i}}$ es el campo radial y $P_{v-1}$ es un polinomio homogéneo de $n$ variables de grado $v-1$.

Más aún, si cualquiera de las condiciones anteriores se cumple, entonces $\operatorname{Sing}\left(\tilde{\mathcal{F}}_{z}\right)$ es el conjunto de puntos $\left[z_{1}, \ldots, z_{n}\right] \in D ;$ los cuales son soluciones del siguiente sistema de $\frac{n(n-1)}{2}+1$ ecuaciones homogéneas:

$$
\mid \begin{aligned}
& P_{v-1}(Z)=0 \\
& z_{j} Z_{i}^{v+1}(z)-z_{i} Z_{j}^{v+1}(z)=0, \quad 1 \leq i<j \leq n
\end{aligned}
$$

Se sigue que $\operatorname{Sing}\left(\tilde{\mathcal{F}}_{z}\right)=\varnothing$ si y sólo si $z=0 \in \mathbb{C}^{n}$ es la única solución del sistema (2).

Denotaremos por $\mathcal{D}_{v}^{n}$ al conjunto de todos los campos vectoriales holomorfos $Z$ definido en $U$ tales que $0 \in \mathbb{C}^{n}$ es la única singularidad dicrítica de $Z$ un $U$, $m_{0}(Z)=v$ y $\operatorname{Sing}\left(\tilde{\mathcal{F}}_{z}\right)=\varnothing$. Observe que los elementos de $\mathcal{D}_{v}^{n}$ son los campos vectoriales holomorfos resueltos después de un "blow-up". Este es un caso particular del Problema de Desingularización el cual fue resuelto por $A$. Seidenberg [16] (cuando $n=2$ ), C. Camacho, F. Cano y P. Sad [7] (en el caso que $0 \in \mathbb{C}^{n}$ es una singularidad absolutamente aislada no dicrítica) y R. Benazic [3] (cuando $0 \in \mathbb{C}^{n}$ es una singularidad absolutamente aislada). Debemos mencionar que una estrategia para el caso general en dimensión compleja 3 fue desarrollada por F. Cano [8], sin embargo, en dimensión $n$ el problema aún no ha sido resuelto.

Para cada $Z \in \mathcal{D}_{v}^{n}, Z=P_{v-1} R+\sum_{k \geq v+1} Z^{k}$, definimos la hipersuperficie algebraica $S$ sobre el divisor $D=\mathbb{C} P\left(\begin{array}{l}k \geq v+1 \\ n-1\end{array}\right)$ como:

$$
S=\left\{\left[z_{1}, \ldots, z_{n}\right] \in D: P_{v-1}\left(z_{1}, \ldots, z_{n}\right)=0\right\}
$$

Sea $p$ cualquier punto del divisor $D$ y $\tilde{L}$ la hoja de $\tilde{\mathcal{F}}_{z}$ que pasa por $p$. En la sección 2 probaremos que si $p \notin S$ entonces $\tilde{L}$ es transversal a $D$. Por eșta razón, llamamos a $S$ la hipersuperficie de tangencia de la foliación $\tilde{\mathcal{F}}_{z}$. Cuando $p \in S$ podemos definir el índice de intersección $i_{p}(D, L)$, el conjunto de tangencia de orden $k \Gamma_{k}(\tilde{Z})$ y las hipersuperficies algebraicas: 


$$
S^{(k)}=\left\{\left[z_{1} ; \ldots ; z_{n}\right] \in D: G_{k}\left(z_{1}, \ldots, z_{n}\right)=0\right\}
$$

donde $G_{k}=Z_{k}^{v+1}\left[P_{v-1}\right]$. En la sección 3 probaremos que el índice de intersección de la hoja $\tilde{L}$ con el divisor $D$ en el punto $p$ es $k+1$ si y sólo si $p \in\left(S \cap S^{(1)} \cap \ldots \cap S^{(k-1)}\right)-S^{(k)}$ (ver el corolario a la Proposición 2.2).

El presente trabajo esta organizado de la manera siguiente: en la sección 2 hacemos los cálculos necesarios para establecer el índice de intersección de las hojas del transformado estricto de $Z$ con el divisor $D$. En la sección 3, consideramos campos vectoriales holomorfos en dimensión 3 y probaremos que si $Z \in \mathcal{D}_{v}^{3}$ tal que si $S$ es no singular y transversal a $S^{(1)}$ entonces el índice de intersección es 1, 2 o 3. Más aún, esta es una condición genérica, es decir, la propiedad mencionada es satisfecha por un conjunto abierto y denso en el espacio normado de campos vectoriales holomorfos (ver Teorema 3.4). En la sección 3, definimos la Condición $L$ y probamos que es una condición genérica en $\mathcal{D}_{v}^{3}$ (ver Teorema 4.2) y finalmente, en el Teorema 4.4 probamos que cualquier par de elementos de $\mathcal{D}_{v}^{3}$ que satisface la Condición L son topológicamente equivalentes, obteniéndose de esta manera, formas normales bastantes simples.

\section{EL INDICE DE INTERSECCIÓN Y EL ORDEN DE TANGENCIA}

Sea $Z \in \mathcal{D}_{v}^{n}, Z=P_{v-1} R+\sum_{k \geq v+1} Z^{k}$, sabemos que cualquier punto $p \in D$ es un punto regular de la foliación $\tilde{\mathcal{F}}_{z}$. Sea $\tilde{L}$ la hoja de $\mathcal{F}_{Z}$ que pasa por $p$. Desde que $D$ y $\tilde{L}$ son subvariedades holomorfas de dimensiones complementarias en $\tilde{U}$ y $p \in D \cap \tilde{L}$, podemos definir el indice de intersección $i_{p}(D, \tilde{L})$ de la hoja $\tilde{L}$ con el divisor $D$ en el punto $p$. En efecto, podemos suponer, sin pérdida de generalidad, que $p$ esta en la carta de $\tilde{U}$ tal que, el "blow-up" $E$ tiene la expresión $E\left(y_{1}, \ldots, y_{n}\right)=\left(y_{1} y_{n}, \ldots, y_{n-1} y_{n}, y_{n}\right)=\left(z_{1}, \ldots, z_{n}\right)$. Un fácil cálculo prueba que el transformado estricto $\mathcal{F}_{Z}$ es generado por el campo vectorial holomorfo $\tilde{Z}=\sum_{i=1}^{n} \tilde{Z}_{i} \frac{\partial}{\partial y_{i}}$, donde

$$
\mid \begin{aligned}
& \tilde{Z}_{i}\left(y_{1}, \ldots, y_{n}\right)=\sum_{k \geq v} y_{n}^{k-v}\left[Z_{i}^{k+1}(\hat{y})-y_{i} Z_{n}^{k+1}(\hat{y})\right], \quad 1 \leq i \leq n-1 \\
& \tilde{Z}_{n}\left(y_{1}, \ldots, y_{n}\right)=P_{v-1}(\hat{y})+\sum_{k \geq v+1} y_{n}^{k-1} Z_{n}^{k}(\hat{y})
\end{aligned}
$$


siendo $\hat{y}=\left(y_{1}, \ldots, y_{n-1}, 1\right)$. Desde que, $p=\left(y_{1}^{0}, \ldots, y_{n-1}^{0}, 0\right)$ es un punto regular de $\tilde{\mathcal{F}}_{z}$, la hoja $\tilde{L}$ puede ser localmente parametrizada por la función holomorfa $\alpha=\left(\alpha_{1}, \ldots, \alpha_{n}\right):\left(\mathbb{D}_{\varepsilon}, 0\right) \rightarrow(\tilde{L}, p)$, donde $\mathbb{D}_{\varepsilon}=\{T \in \mathbb{C}:|T|<\varepsilon\}$, tal que

$$
\mid \begin{aligned}
& \alpha_{i}^{\prime}(T)=\tilde{Z}_{i}(\alpha(T)), \quad \forall 1 \leq i \leq n, \forall T \in \mathbb{D}_{\varepsilon} \\
& \alpha(0)=p .
\end{aligned}
$$

en donde $\tilde{Z}=\sum_{i=1}^{n} \tilde{Z}_{i} \frac{\partial}{\partial y_{i}}$ es el campo vectorial holomorfo que genera la foliación $\tilde{\mathcal{F}}_{z}$. Desde que, $D=\left\{y_{n}=0\right\}$, podemos definir

$$
i_{p}(D, \tilde{L})=\operatorname{ord}_{0}\left(\alpha_{n}\right)
$$

es decir, $i_{p}(D, \tilde{L})=m$ si y sólo si $\alpha_{n}(T)=T^{m} \xi_{n}(T)$ siendo $\xi_{n}$ una función holomorfa tal que $\xi_{n}(0) \neq 0$. Es claro que, la definición anterior es independiente de la elección del camino holomorfo $\alpha$ y que $i_{p}(D, \tilde{L}) \geq 1, \forall p \in D$. Este índice de intersección es un invariante topológico (ver [4]) y puede ser geométricamente interpretado como el número de puntos de intersección de $\tilde{L}$ con una pequeña traslación del divisor $\left\{y_{n}=\delta\right\}$, con $\delta \neq 0$. Para mayor información sobre las propiedades del índice de intersección entre subvariedades complejas de dimensiones complementarias, el lector debe consultar [11].

Existe otra definición del índice de intersección la cual proviene de generalizar a variedades complejas los trabajos de P. Percell [15] y M. I Camacho [9] hechos en variedades reales. En efecto, en primer lugar es fácil ver que es posible encontrar una función $\beta$ sobre $U$ tal que
a) $\beta^{-1}(0)=D$
b) $0 \in \mathbb{C}$ es un valor regular de $\beta$.

Dado $\tilde{Z}=\sum_{i=1}^{n} \tilde{Z}_{i} \frac{\partial}{\partial y_{i}}$, definimos:
a) $\tilde{Z}^{0} \beta=\beta$,
b) $\tilde{Z} \beta=\sum_{i=1}^{n} \tilde{Z}_{i} \frac{\partial \beta}{\partial_{i}}$,
c) $\quad \tilde{Z}^{k+1} \beta(k \geq 1)$ es definido inductivamente por $\tilde{Z}\left(\tilde{Z}^{k} \beta\right)$. 
Definición 2.1 Sea $\beta$ una función que satisface (8) y suponga que $Z \in \mathcal{D}_{v}^{n}$. Para $k \in \mathbb{Z}^{+}$, definimos el conjunto de tangencia de orden $k, \Gamma_{k}(\tilde{Z})$, como el conjunto de todos los puntos $p \in D$ tales que

$$
\beta(p)=(\tilde{Z} \beta)(p)=\ldots=\left(\tilde{Z}^{k-1} \beta\right)(p)=0 \quad \text { y }\left(\tilde{Z}^{k} \beta\right)(p) \neq 0 .
$$

Es fácil ver que los conjuntos $\Gamma_{k}(\tilde{Z})$ son independientes de la elección de la función $\beta$ que cumpla la condición (8). El siguiente resultado justifica porque el conjunto $\Gamma_{k}(\tilde{Z})$ es llamado conjunto de tangencia de orden $k$.

Proposición 2.1 Con las notaciones anteriores, tenemos que

$$
p \in \Gamma_{k}(\tilde{Z}) \Leftrightarrow i_{p}(D, \tilde{L})=k
$$

Demostración. Trabajando en la carta $\tilde{U}$ tal que el "blow-up" se expresa como

$$
E\left(y_{1}, \ldots, y_{n}\right)=\left(y_{1} y_{n}, \ldots, y_{n-1} y_{n}, y_{n}\right)
$$

el divisor es dado por $D=\left\{y_{n}=0\right\}$, por lo tanto podemos considerar $\beta\left(y_{1}, \ldots, y_{n}\right)=y_{n}$. Sea $p \in D$ y $\tilde{L}$ la hoja $\tilde{\mathcal{F}}_{z}$ que pasa por $p$, para $\alpha$ definido como en (6), tenemos que $\alpha_{n}(T)=\beta(\alpha(T))=\left(\tilde{Z}^{0} \beta\right)(\alpha(T))$. Procediendo por inducción, suponga que $\alpha_{n}^{(k)}(T)=\left(\tilde{Z}^{k} \beta\right)(\alpha(T))$, entonces

$$
\begin{aligned}
\alpha_{n}^{(k+1)}(T) & =\sum_{i=1}^{n} \frac{\partial\left(\tilde{Z}^{k} \beta\right)}{\partial y_{i}}(\alpha(T)) \alpha_{i}^{\prime}(T) \\
& =\sum_{i=1}^{n} \frac{\partial\left(\tilde{Z}^{k} \beta\right)}{\partial y_{i}}(\alpha(T)) \tilde{Z}_{i}(\alpha(T)) \\
& =\left(\tilde{Z}^{k+1} \beta\right)(\alpha(T))
\end{aligned}
$$

Concluimos que $\alpha_{n}^{(k)}(0)=\left(\tilde{Z}^{k} \beta\right)(p), \forall k \in \mathbb{N}$. Luego

$$
\begin{aligned}
p \in \Gamma_{k}(\tilde{Z}) & \Leftrightarrow\left(\tilde{Z}^{j} \beta\right)(p)=0(0 \leq j \leq k-1) \text { y }\left(\tilde{Z}^{j} \beta\right)(p) \neq 0 \\
& \Leftrightarrow \alpha_{n}^{(j)}(0)=0(0 \leq j \leq k-1) \text { y } \alpha_{n}^{(k)}(0) \neq 0 \\
& \Leftrightarrow i_{p}(D, \tilde{L})=k
\end{aligned}
$$

Esto prueba la proposición. 
Los conjuntos $\Gamma_{k}(\tilde{Z})(k \in \mathbb{N})$ forman una partición del divisor $D$. Observe que dado $p=\left(y_{1}^{0}, \ldots, y_{n-1}^{0}, 0\right) \in D$, de (5) y (6) tenemos que $\alpha_{n}^{\prime}(0)=P_{v-1}\left(y_{1}^{0}, \ldots, y_{n-1}^{0}, 0\right)$. Por lo tanto, $i_{p}(D, \tilde{L})=1 \Leftrightarrow p \in D-S \Leftrightarrow \tilde{L}$ es transversal a $D$. Concluimos que $\Gamma_{1}(\tilde{Z})=D-S$.

Para $p \in S$, vamos a calcular $\alpha_{n}^{\prime \prime}(0)$. De (5) y (6),

$$
\alpha_{n}^{\prime \prime}(T)=\sum_{i=1}^{n} \frac{\partial \tilde{Z}_{n}}{\partial y_{i}}(\alpha(T)) \alpha_{i}^{\prime}(T)
$$

luego

$$
\begin{aligned}
\alpha_{n}^{\prime \prime}(0) & =\sum_{i=1}^{n-1} \frac{\partial \tilde{Z}_{n}}{\partial y_{i}}(p) \tilde{Z}_{i}(p) \\
& =\sum_{i=1}^{n-1} \frac{\partial \tilde{P}_{v-1}}{\partial z_{i}}\left(\tilde{y}_{0}\right)\left[\tilde{Z}_{i}^{v+1}\left(\tilde{y}_{0}\right)-y_{i}^{0} \tilde{Z}_{n}^{v+1}\left(\tilde{y}_{0}\right)\right] \\
& =\sum_{i=1}^{n-1} \frac{\partial \tilde{P}_{v-1}}{\partial z_{i}}\left(\tilde{y}_{0}\right) \tilde{Z}_{i}^{v+1}\left(\tilde{y}_{0}\right)-\tilde{Z}_{n}^{v+1}\left(\tilde{y}_{0}\right) \sum_{i=1}^{n-1} y_{i}^{0} \frac{\partial \tilde{P}_{v-1}}{\partial z_{i}}\left(\tilde{y}_{0}\right)
\end{aligned}
$$

donde $\tilde{y}_{0}=\left(y_{1}^{0}, \ldots, y_{n-1}^{0}, 1\right)$. De esta manera

$$
\alpha_{n}^{\prime \prime}(0)=\sum_{i=1}^{n-1} \frac{\partial \tilde{P}_{v-1}}{\partial z_{i}}\left(y_{1}^{0}, \ldots, y_{n-1}^{0}, 1\right) \tilde{Z}_{i}^{v+1}\left(y_{1}^{0}, \ldots, y_{n-1}^{0}, 1\right) .
$$

Definimos el polinomio homogéneo $Q_{2 v-1}$ de grado $2 v-1$ como

$$
Q_{2 v-1}=Z^{v+1}\left[P_{v-1}\right]=\sum_{i=1}^{n-1} \frac{\partial P_{v-1}}{\partial z_{i}} Z_{i}^{v+1}
$$

y consideramos la hipersuperficie algebraica

$$
S^{(1)}=\left\{\left[z_{1} ; \ldots ; z_{n}\right] \in D: Q_{2 v-1}\left(z_{1}, \ldots, z_{n}\right)=0\right\}
$$

de (10) deducimos que $i_{p}(D, \tilde{L})=2 \Leftrightarrow p \in S-S^{(1)}$, es decir $\Gamma_{2}(\tilde{Z})=S-S^{(1)}$ y $\Gamma_{3}(\tilde{Z}) \subseteq S-S^{(1)}$. Note que los puntos singulares de la hipersuperficie de tangencia $S$ están contenidos en $S^{(1)}$. Sin embargo $S \cap S^{(1)}$ contiene otros puntos $p$ los cuales no son singularidades de $S$ pero $i_{p}(D, \tilde{L})>2$. 
Dado $k \in \mathbb{N}$, definimos los polinomios homogéneos $G_{k}$ de grado $(k+1) v-1$ como $G_{k}=Z_{k}^{v+1}\left[P_{v-1}\right]$ y consideramos las hipersuperficies algebraicas

$$
S^{(k)}=\left\{\left[z_{1} ; \ldots ; z_{n}\right] \in D: G_{k}\left(z_{1}, \ldots, z_{n}\right)=0\right\}
$$

Observe que, en particular, $S^{(0)}=S, G_{0}=P_{v-1}$ y $G_{1}=Q_{2 v-1}$.

Sea $Z=\sum_{k \geq v} Z_{k}$ un elemento de $\mathcal{D}_{v}^{n}$, donde $Z_{v}=P_{v-1} R$ y $Z_{k}=\sum_{i=1}^{n} Z_{i}^{k} \frac{\partial}{\partial z_{i}}$. En coordenadas $y_{1}=\frac{z_{1}}{z_{n}}, \ldots, y_{n-1}=\frac{z_{n-1}}{z_{n}}, y_{n}=z_{n} ;$ tenemos que $\tilde{Z}=\tilde{Z}_{v}+\tilde{Z}_{v+1}+y_{n} \tilde{Y}$, donde $Z_{v}=P_{v-1}(\hat{y}) \frac{\partial}{\partial y_{n}}, \tilde{Z}_{v+1}=\sum_{i=1}^{n-1}\left[Z_{i}^{v+1}(\hat{y})-y_{i} Z_{n}^{v+1}(\hat{y})\right] \frac{\partial}{\partial y_{i}}+y_{n} Z_{n}^{v+1}(\hat{y}) \frac{\partial}{\partial y_{n}}$, donde $\hat{y}=\left(y_{1}, \ldots, y_{n-1}, 1\right)$ e $\hat{Y}=\sum_{k \geq v+2} y_{n}^{k-v-2} \tilde{Z}^{k}$. Más aún, en estas coordenadas, la ecuación afín de $S^{(k)}$ es dada por

$$
S^{(k)}=\left\{\left(y_{1}, \ldots, y_{n-1}\right) \in \mathbb{C}^{n-1}: g_{k}\left(y_{1}, \ldots, y_{n-1}\right)=0\right\}
$$

donde $g_{k}\left(y_{1}, \ldots, y_{n-1}\right)=G_{k}\left(y_{1}, \ldots, y_{n-1}, 1\right)=G_{k}(\hat{y})$. Observe que $\left(\tilde{Z}^{v} \beta\right)(y)=G_{0}(\hat{y})$.

Lema 2.1 Con las notaciones anteriores, para cualquier $k \in \mathbb{Z}^{+}$tenemos que

$$
\left(\tilde{Z}_{k}^{v+1} \tilde{Z}^{v} \beta\right)(y)=G_{k}(\hat{y})+\sum_{r=0}^{k-1} f_{k, r}(\hat{y}) G_{r}(\hat{y})
$$

donde $f_{k, r}: \mathbb{C}^{n} \rightarrow \mathbb{C}$ son funciones holomorfas.

Demostración. Primeramente, observamos que para cualquier $r \in \mathbb{N}$ se tiene

$$
\begin{aligned}
\tilde{Z}^{v+1}\left[G_{r}(\hat{y})\right] & =\sum_{i=1}^{n-1}\left[Z_{i}^{v+1}(\hat{y})-y_{i} Z_{n}^{v+1}(\hat{y})\right] \frac{\partial G_{r}}{\partial z_{i}}(\hat{y}) \\
& =\sum_{i=1}^{n-1} Z_{i}^{v+1}(\hat{y}) \frac{\partial G_{r}}{\partial z_{i}}(\hat{y})-Z_{n}^{v+1}(\hat{y}) \sum_{i=1}^{n-1} y_{i} \frac{\partial G_{r}}{\partial z_{i}}(\hat{y}) \\
& =\sum_{i=1}^{n-1} Z_{i}^{v+1}(\hat{y}) \frac{\partial G_{r}}{\partial z_{i}}(\hat{y})-Z_{n}^{v+1}(\hat{y})\left[((r+1) v-1) G_{r}(\hat{y})-\frac{\partial G_{r}}{\partial z_{i}}(\hat{y})\right] \\
& =\sum_{i=1}^{n-1} Z_{i}^{v+1}(\hat{y}) \frac{\partial G_{r}}{\partial z_{i}}(\hat{y})-((r+1) v-1) Z_{n}^{v+1}(\hat{y}) G_{r}(\hat{y}) \\
& =\left(Z^{v+1} G_{r}\right)(\hat{y})+\phi_{r}(\hat{y}) G_{r}(\hat{y})
\end{aligned}
$$


donde $\phi_{r}=((r+1) v-1) Z_{n}^{v+1}$. Por lo tanto,

$$
\tilde{Z}^{v+1}\left[G_{r}(\hat{y})\right]=G_{r+1}(\hat{y})+\phi_{r}(\hat{y}) G_{r}(\hat{y})
$$

Ahora, por inducción, suponga que

$$
\left(Z_{v+1}^{k-1} \tilde{Z}_{v} \beta\right)(y)=G_{k-1}(\hat{y})+\sum_{r=0}^{k-2} f_{k-1, r}(\hat{y}) G_{r}(\hat{y})
$$

De (15), tenemos que

$$
\begin{aligned}
\left(\tilde{Z}_{v+1}^{k} \tilde{Z}_{v} \beta\right)(y) & =\tilde{Z}_{v+1}\left[G_{k-1}(\hat{y})\right]+ \\
& =+\sum_{r=0}^{k-2}\left[\tilde{Z}_{v+1}\left[f_{k-1, r}(\hat{y})\right] G_{r}(\hat{y})+f_{k-1, r}(\hat{y}) \tilde{Z}_{v+1}\left[G_{r}(\hat{y})\right]\right] \\
& =G_{r}(\hat{y})+\phi_{k-1}(\hat{y}) G_{k-1}(\hat{y})+\sum_{r=0}^{k-2} \tilde{Z}_{v+1}\left[f_{k-1, r}(\hat{y})\right] G_{r}(\hat{y})+ \\
& =\sum_{r=0}^{k-2} f_{k-1, r}(\hat{y})\left[G_{k+1}(\hat{y})+\phi_{r}(\hat{y}) G_{r}(\hat{y})\right]
\end{aligned}
$$

Denotado $f_{k, k-1}=\phi_{k-1}+f_{k-1, k-2}, f_{k, r}=\tilde{Z}_{v+1}\left[f_{k-1, r}(\hat{y})\right]+f_{k-1, r-1}+f_{k-r, r} \phi_{r},(1 \leq r \leq k-2) \mathrm{y}$ $f_{k, 0}=\phi_{0}$, la proposición esta probada.

Corolario. Sea $r$ un entero no negativo y $p=\left(y_{1}^{0}, \ldots, y_{n-1}^{0}, 0\right) \in S^{(r)}$. Entonces,

$$
\tilde{Z}_{v+1}\left[g_{r}\right](p)=\left(Z_{v+1} G_{r}\right)\left(y_{1}^{0}, \ldots, y_{n-1}^{0}, 1\right)
$$

Lema 2.2 Para cualquier $k \in \mathbb{Z}^{+}$, tenemos que

$$
\tilde{Z}^{k} \beta=\tilde{Z}_{v+1}^{k-1} \tilde{Z}_{v} \beta+\sum_{j=0}^{k-2} \tilde{Z}_{v+1}^{j} \tilde{Z}_{v} \beta \cdot F_{k, j+1}+y_{n} F_{k, k}
$$

donde $F_{k, j}: \mathbb{C}^{n} \rightarrow \mathbb{C}$ son funciones analíticas.

Demostración. Desde que $\tilde{Z}=\tilde{Z}_{v}+\tilde{Z}_{v+1}+y_{n} \hat{Y}$, para cualquier $\tilde{f}: \mathbb{C}^{n} \rightarrow \mathbb{C}$, tenemos

$$
\tilde{Z} \tilde{f}=\tilde{Z}_{v} \tilde{f}+\tilde{Z}_{v+1} \tilde{f}+y_{n} \tilde{Y} \tilde{f}=\tilde{Z}_{1} \beta \frac{\partial \tilde{f}}{\partial y_{n}}+\tilde{Z}_{v+1} \tilde{f}+y_{n} \tilde{Y} \tilde{f}
$$


Por lo tanto $\tilde{Z} \beta=\tilde{Z}_{v} \beta+\tilde{Z}_{v+1} \beta+y_{n} \hat{Y} \beta=\tilde{Z}_{v} \beta+y_{n} F_{1,1}$, donde $F_{1,1}=A_{v+1}^{n}(\hat{y})+\tilde{Y} \beta$. Ahora, suponga por inducción que

$$
Z^{k-1} \beta=\tilde{Z}_{v+1}^{k-2} \tilde{Z}_{v} \beta+\sum_{j=0}^{k-3} \tilde{Z}_{v+1}^{j} \tilde{Z}_{v} \beta \cdot F_{k-1, j+1}+y_{n} F_{k-1, k-1}
$$

De (16) y (17), tenemos

$$
\begin{aligned}
\tilde{Z}^{k} \beta= & \tilde{Z}\left(\tilde{Z}_{v+1}^{k-2} \tilde{Z}_{v} \beta\right)+\sum_{j=0}^{k-3} \tilde{Z}\left(\tilde{Z}_{v+1}^{j} \tilde{Z}_{v} \beta\right) \cdot F_{k-1, j+1} \\
& +\sum_{j=0}^{k-3} \tilde{Z}_{v+1}^{j} \tilde{Z}_{v} \beta \cdot \tilde{Z}\left(F_{k-1, j+1}\right)+y_{n} \tilde{Z}\left(F_{k-1, k-1}\right)+\tilde{Z} \beta \cdot F_{k-1, k-1} \\
= & \tilde{Z}_{v} \beta \frac{\partial\left(Z_{v+1}^{k-2} \tilde{Z}_{v} \beta\right)}{\partial y_{n}}+Z_{v+1}^{k-1} \tilde{Z}_{v} \beta+y_{n} \tilde{Y} Z_{v+1}^{k-2} \tilde{Z}_{v} \beta \\
& +\sum_{j=0}^{k-3}\left[\tilde{Z}_{v} \beta \frac{\partial\left(Z_{v+1}^{j} \tilde{Z}_{v} \beta\right)}{\partial y_{n}}+Z_{v+1}^{i+1} \tilde{Z}_{v} \beta+y_{n} \tilde{Y} Z_{v+1}^{j} \tilde{Z}_{v} \beta\right] F_{k-1, j+1} \\
& +\sum_{j=0}^{k-3} \tilde{Z}_{v+1}^{j} \tilde{Z}_{v} \beta \cdot \tilde{Z}\left(F_{k-1, j+1}\right)+y_{n} \tilde{Z}\left(F_{k-1, k-1}\right)+ \\
& +\left[\tilde{Z}_{v} \beta+y_{n} F_{1,1}\right] F_{k-1, k-1}
\end{aligned}
$$

\section{Denotando}

$$
\begin{aligned}
F_{k, 1}= & \tilde{Z}\left(F_{k-1,1}\right)+\frac{\partial\left(\tilde{Z}_{v+1}^{k-2} \tilde{Z}_{v} \beta\right)}{\partial y_{n}}+ \\
& +\sum_{j=0}^{k-3} \frac{\partial\left(\tilde{Z}_{v+1}^{j} \tilde{Z}_{v} \beta\right)}{\partial y_{n}} F_{k-1, j+1}+F_{k-1, k-1} \\
F_{k, j+1}= & F_{k-1, j}+\tilde{Z}\left(F_{k-1, j+1}\right), \quad 1 \leq j \leq k-3 \\
F_{k, k-1}= & F_{k-1, k-2} \\
\mathrm{y} \quad & \\
F_{k, k}= & \tilde{Y} \tilde{Z}_{v+1}^{k-2} \tilde{Z}_{v} \beta+\sum_{j=0}^{k-3} \tilde{Y} \tilde{Z}_{v+1}^{j} \tilde{Z}_{v} \beta \cdot F_{k-1, j+1}+ \\
& +\tilde{Z}\left(F_{k-1, k-1}\right)+F_{1,1} F_{k-1, k-1},
\end{aligned}
$$

se sigue la prueba de la proposición. 
Corolario. Para cualquier $k \in \mathbb{Z}^{+}$, existen funciones analíticas

$$
H_{k, 1}, \ldots, H_{k, n}: \mathbb{C}^{n} \rightarrow \mathbb{C}
$$

Tales que

$$
\left(\tilde{Z}^{k} \beta\right)(y)=G_{k-1}(\hat{y})+\sum_{j=0}^{k-2} H_{k, k-j-1}(y) G_{j}(\hat{y})+y_{n} H_{k, k}(y)
$$

Proposición 2.2 Sea $k$ un entero positivo, $p \in \bigcap_{j=0}^{k-1} S^{(j)}$. Se cumple

$$
i_{p}(D, \tilde{L})=k+1 \Leftrightarrow p \notin S^{(k)}
$$

Demostración. Supongamos que $p=\left(y_{1}^{0}, \ldots, y_{n-1}^{0}, 0\right)$. Como

$$
p \in S \cap S^{(1)} \cap \ldots \cap S^{(k-1)},
$$

tenemos que $G_{j}\left(y_{1}^{0}, \ldots, y_{n-1}^{0}, 1\right)=0, \forall 1 \leq j \leq k-1$. Por la proposición 2.1 y el Lema 2.2 tenemos

$$
\begin{aligned}
\alpha_{n}^{(k+1)}(0)= & \left(\tilde{Z}^{k+1} \beta\right)(p)=\left(\tilde{Z}_{v+1}^{k} \tilde{Z}_{v} \beta\right)(p)+ \\
& +\sum_{j=0}^{k-1}\left(\tilde{Z}_{v+1}^{j} \tilde{Z}_{v} \beta\right)(p) F_{k, j+1}(p)
\end{aligned}
$$

Por el Lema 2.1, tenemos

$$
\begin{aligned}
\left(\tilde{Z}_{v+1}^{j} \tilde{Z}_{v} \beta\right)(p)= & G_{j}\left(y_{1}^{0}, \ldots, y_{n-1}^{0}, 1\right) \\
& +\sum_{r=0}^{j-1} f_{k, r}\left(y_{1}^{0}, \ldots, y_{n-1}^{0}, 1\right) G_{r}\left(y_{1}^{0}, \ldots, y_{n-1}^{0}, 1\right)
\end{aligned}
$$

De (18) and (19), se sigue que $\alpha_{n}^{(k+1)}(0)=G_{k}\left(y_{1}^{0}, \ldots, y_{n-1}^{0}, 1\right)$. Esta igualdad implica la Proposición.

Corolario Para cualquier $Z \in \mathcal{D}_{v}^{n}$ tenemos

$$
\Gamma_{k+1}(\tilde{Z})=\left(\bigcap_{j=0}^{k-1} S^{(j)}\right)-S^{(k)}, \forall k \in \mathbb{Z}^{+}
$$




\section{LOS CONJUNTOS DE TANGENCIA PARA CAMPOS VECTORIALES} EN $\mathcal{D}_{v}^{3}$

Sea $Z \in \mathcal{D}_{v}^{3}$ un campo vectorial holomorfo tal que $Z=P_{v-1} R+\sum_{k \geq v+1} Z_{k} \mathrm{y}$ $Z_{k}=\sum_{i=1}^{3} A_{k}^{i} \frac{\partial}{\partial z_{i}}$. En este caso, las hipersuperficies $S^{(k)}(k \in \mathbb{N})$ son curvas algebraicas sobre $D=\mathbb{C} P(2)$.

Observación. $S$ y $S^{(1)}$ no tienen componentes comunes, por lo tanto

$$
\#\left(S \cap S^{(1)}\right)<+\infty
$$

(ver [5]).

En esta sección probaremos que genéricamente $\Gamma_{k}(\tilde{Z})=\emptyset, \forall k \geq 4$ es decir $D=\bigcup_{j=1}^{3} \Gamma_{j}(\tilde{Z})$.

Proposición 3.1 Si $P_{v-1}$ es un polinomio homogéneo irreducible sin puntos singulares y $p \in S \cap S^{(1)}$, entonces

$$
i_{p}(D, \tilde{L})=3 \text { si y sólo si } S \text { es transversal a } S^{(1)} \text { en } p
$$

Demostración. Sea $p=\left(y_{1}^{0}, y_{2}^{0}, 0\right)$. De la Proposición y el Corolario del Lema, tenemos

$$
\begin{aligned}
\alpha_{3}^{\prime \prime \prime}(0) & =G_{2}\left(y_{1}^{0}, y_{2}^{0}, 1\right)=\left(Z_{v+1} G_{1}\right)\left(y_{1}^{0}, y_{2}^{0}, 1\right)=\tilde{Z}_{v+1}\left[g_{1}\right](p) \\
& =\sum_{i=1}^{2}\left[Z_{v+1}^{i}\left(y_{1}^{0}, y_{2}^{0}, 1\right)-y_{i}^{0} Z_{v+1}^{3}\left(y_{1}^{0}, y_{2}^{0}, 1\right)\right] \frac{\partial g_{1}}{\partial y_{i}}\left(y_{1}^{0}, y_{2}^{0}\right)
\end{aligned}
$$

Por otro lado, desde que $p \in S^{(1)}$ podemos obtener

$$
\begin{aligned}
0 & =G_{1}\left(y_{1}^{0}, y_{2}^{0}, 1\right)=\left(Z_{v+1} G_{0}\right)\left(y_{1}^{0}, y_{2}^{0}, 1\right)=\tilde{Z}_{v+1}\left[g_{0}\right][p] \\
& =\sum_{i=1}^{2}\left[Z_{v+1}^{i}\left(y_{1}^{0}, y_{2}^{0}, 1\right)-y_{i}^{0} Z_{v+1}^{3}\left(y_{1}^{0}, y_{2}^{0}, 1\right)\right] \frac{\partial g_{0}}{\partial y_{i}}\left(y_{1}^{0}, y_{2}^{0}\right)
\end{aligned}
$$

Denotado $a_{i}=Z_{v+1}^{i}\left(y_{1}^{0}, y_{2}^{0}, 1\right)-y_{i}^{0} Z_{v+1}^{3}\left(y_{1}^{0}, y_{2}^{0}, 1\right) \quad$ y $\quad c_{i j}=\frac{\partial g_{j-1}}{\partial y_{i}}\left(y_{1}^{0}, y_{2}^{0}\right)$ donde $1 \leq i, j \leq 2$, de (20) y (21) tenemos 


$$
\alpha_{3}^{\prime \prime \prime}(0)=a_{1} c_{12}+a_{2} c_{22} \quad \text { y } a_{1} c_{11}+a_{2} c_{21}=0
$$

Como $p$ es un punto regular de la curva algebraica $S$, podemos suponer sin pérdida de generalidad que $c_{11} \neq 0$. Bajo esta suposición observamos que $a_{2} \neq 0$. Por lo tanto

$$
c_{11} \alpha_{3}^{\prime \prime \prime}(0)=a_{1} c_{11} c_{12}+a_{2} c_{11} c_{22}=a_{2} c_{21} c_{12}+a_{2} c_{11} c_{21}=a_{2} \operatorname{det}\left(c_{i j}\right)
$$

Por lo tanto

$$
\alpha_{3}^{\prime \prime \prime}(0)=\frac{a_{2}}{c_{11}} \operatorname{det}\left(c_{i j}\right)
$$

Ahora, $S$ es transversal a $S^{(1)}$ en el punto $p$ si y sólo si,

$$
\left(\nabla g_{i}\right)\left(y_{1}^{0}, y_{2}^{0}\right)=\left(\frac{\partial g_{i}}{\partial y_{1}}\left(y_{1}^{0}, y_{2}^{0}\right), \frac{\partial g_{i}}{\partial y_{2}}\left(y_{1}^{0}, y_{2}^{0}\right)\right), \quad(i=1,2)
$$

son vectores linealmente independientes y esto es equivalente a decir que $\operatorname{det}\left(c_{i j}\right) \neq 0$. De esta observación y de (22), la prueba de la proposición se sigue inmediatamente.

Corolario. Suponga que $P_{v-1}$ es un polinomio homogéneo irreducible sin puntos singulares y que $p \in S \cap S^{(1)}$. Si $S$ es transversal a $S^{(1)}$ entonces

$$
p \in S \cap S^{(1)} \Rightarrow p \in D-S^{(2)}
$$

Más específicamente, $\Gamma_{k}(\tilde{Z})=\emptyset, \forall k \geq 4$.

Ahora, suponga que $Z=P_{v-1} R+\sum_{k \geq v+1} Z_{k} \in \mathcal{D}_{v}^{3}$ es un campo vectorial holomorfo tal que $S$ es una curva algebraica irreducible no singular y $S^{(1)}$ no es transversal a $S$. Bajo estas condiciones, podemos encontrar un $Z_{\varepsilon} \in \mathcal{D}_{v}^{3}$ suficientemente próximo a $Z$ (en la norma del espacio Campos Vectoriales) tal que $S_{\varepsilon}$. y $S_{\varepsilon}^{(1)}$ son transversales. En efecto, para $\varepsilon>0$ definimos $Z_{\varepsilon}=Z+\varepsilon Y_{v+1}$, donde $Y_{v+1}$ es un campo vectorial homogéneo de grado $v+1$. Observe que $S_{\varepsilon}=S$ y $S_{\varepsilon}^{(1)}=\left(G_{1, \varepsilon}=0\right)$ con 


$$
G_{1, \varepsilon}=\left(Z_{v+1}+\varepsilon Y_{v+1}\right)\left[P_{v-1}\right]=G_{1}+\varepsilon Y_{v+1}\left[P_{v-1}\right]
$$

Se sigue que si $\varepsilon$ es suficientemente pequeño entonces $S$ es transversal a $S_{\varepsilon}^{(1)}$.

Proposición 3.2 Para cualquier $Z \in \mathcal{D}_{v}^{3}$ tal que $S$ es una curva algebraica no singular irreducible, existe $Z_{\varepsilon} \in \mathcal{D}_{v}^{3}$ suficientemente próximo a $Z$ tal que $S_{\varepsilon}=S$ y $S_{\varepsilon}^{(1)}$ es transversal a $S$.

Cuando $P_{v-1}$ no es un polinomio irreducible, podemos escribir $P_{v-1}=F_{1}^{{ }^{r_{1}}} \ldots F_{l}^{r_{i}}$ donde los $F_{j}$ son polinomios homogéneos irreducibles de grado $d_{j}$ y $r_{j}$ es la multiplicidad $F_{j}(1 \leq j \leq l)$. Esta factorización es única salvo constantes. Correspondiente a la factorización anterior, podemos escribir las curvas de tangencias $S=r_{1} S_{1}+\ldots+r_{l} S_{l}$, where $S_{j}=\left(F_{j}=0\right)$ son llamadas las componentes irreducibles de $S$.

Suponga que la curva de tangencia $S$ no tiene componente múltiple, (es decir $\left.r_{1}=\ldots=r_{1}=1\right)$, por las técnicas usuales de la teoría de curvas algebraicas (ver por ejemplo [6]) es posible construir una familia $S_{t}$ de curvas algebraicas en $\mathbb{C P}(2)$ tal que $S_{0}$ es isomorfa a $S S_{t}$ es una curva no singular de grado $v-1$ para $t \neq 0$. En efecto, sea $S_{1}$ la otra curva algebraica en $\mathbb{C} P(2)$ de grado $v-1$ dada por la ecuación $\left(Q_{v-1}=0\right)$ tal que $S_{1}$ a la curva $\tilde{S}$ en $(v-1)^{2}$ puntos distintos (es decir, $S$ es transversal a $S_{1}$ ). Consideremos la familia de curvas a un parámetro $\left\{S_{\left[t_{0} ; i_{1}\right]}\right\}_{\left[t_{0} ; t_{1}\right] \in \mathbb{C} P(1)}$, donde

$$
S_{\left[t_{0} ; t_{1}\right]}\left\{\left[z_{1} ; z_{2} ; z_{3}\right] \in \mathbb{C P}(2): t_{0} P_{v-1}\left(z_{1}, z_{2}, z_{3}\right)+t_{1} Q_{v-1}\left(z_{1}, z_{2}, z_{3}\right)=0\right\}
$$

Note que

$$
\mathbb{C} P(2)=\bigcup_{\left[t_{0} ; t_{1}\right] \in \mathbb{C} P(1)} S_{\left[t_{0} ; t_{1}\right]} \text { y } S \cap S_{I} \subset S_{\left[t_{0} ; t_{1}\right]}, \forall\left[t_{0} ; t_{1}\right] \in \mathbb{C} P(1)
$$

Hacemos explosiones en cada de los $(v-1)^{2}$ puntos de intersección y obtenemos así un mapeo holomorfo $\pi: Y \rightarrow \mathbb{C P}(2)$, donde $Y$ es una variedad algebraica compacta. Definimos $f: Y \rightarrow \mathbb{C P}(1)$ por $f(y)=\left[t_{0} ; t_{1}\right]$ si y sólo si 
$\pi(y) \in S_{\left[t_{0} ; t_{1}\right]}$. Es fácil ver que el mapeo $f$ esta bien definido, es holomorfo y las fibras $f^{-1}\left(\left[t_{0} ; t_{1}\right]\right)$ son isomorfas a $S_{\left[t_{0} ; t_{1}\right]}$. En particular $f^{-1}([1 ; 0])$ es isomorfo a $S$. Del teorema de Bertini (ver [12], o [13]) se sigue que, salvo un número finito de puntos, $\left[t_{0} ; t_{1}\right] \in \mathbb{C} P(1)$, la fibra $f^{-1}\left(\left[t_{0} ; t_{1}\right]\right)$ es singular e irreducible, como ha sido probado.

Para $\varepsilon>0$ consideramos

$$
S_{[1 ; \varepsilon]}=\left\{\left[z_{1} ; z_{2} ; z_{3}\right] \in \mathbb{C} P(2): P_{v-1}\left(z_{1}, z_{2}, z_{3}\right)+\varepsilon Q_{v-1}\left(z_{1}, z_{2}, z_{3}\right)=0\right\},
$$

se sigue que si $\varepsilon$ es suficientemente pequeño entonces $Z_{\varepsilon}=Z+\varepsilon Q_{v-1} R$ es un campo vectorial holomorfo tal que $Z_{\varepsilon} \in \mathcal{D}_{v}^{3}, Z_{\varepsilon}$ esta próximo a $Z$ y $S_{\varepsilon}=S_{[1 ; \varepsilon]}$ es una curva algebraica irreducible no singular.

Finalmente, si $P_{v-1}$ tiene factores múltiples, es decir $P_{v-1}=F_{1}^{r_{1}} \ldots F_{l}^{r_{i}}$ entonces consideramos el polinomio homogéneo

$$
P_{v-1}=\left(\prod_{k=1}^{n_{1}} F_{1, k}\right) \ldots\left(\prod_{k=1}^{r_{1}} F_{l, k}\right),
$$

donde $F_{j, k}=F_{j}+\varepsilon_{j, k} H_{j}, \varepsilon_{j, k}>0$ y $H_{j}$ son polinomios homogéneos de grado $d_{j}\left(1 \leq j \leq r_{l}\right)$. Es posible elegir $\varepsilon_{j, k}$ suficientemente pequeño y un $H_{j}$ conveniente tal que $P_{v-1}$ esté suficientemente próximo de $P_{v-1}$ y $F_{j, k}$ son polinomios homogéneos irreducibles. De esta manera, hemos probado la siguiente

Proposición 3.3 Para cualquier $Z \in \mathcal{D}_{v}^{3}$, existe $Z_{\varepsilon} \in \mathcal{D}_{v}^{3}$ suficientemente próximo de $Z$ tal que $S_{\varepsilon}$ es una curva algebraica no singular.

Finalmente, de las Proposiciones 3.1, 3.2 y 3.3, obtenemos

Teorema 3.4 Para cualquier $Z \in \mathcal{D}_{v}^{3}$, existe $Z_{\varepsilon} \in \mathcal{D}_{v}^{3}$ suficientemente próximo a $Z$ tal que $S_{\varepsilon}$ es una curva algebraica no singular y $S_{\varepsilon}^{(1)}$ es transversal a $S_{\varepsilon}$. Más aún $\Gamma_{1}\left(\tilde{Z}_{\varepsilon}\right)=D-S_{\varepsilon}, \Gamma_{2}\left(\tilde{Z}_{\varepsilon}\right)=S_{\varepsilon}-S_{\varepsilon}^{(1)}, \Gamma_{3}\left(\tilde{Z}_{\varepsilon}\right)=S_{\varepsilon} \cap S_{\varepsilon}^{(1)}$ y $\Gamma_{k}\left(\tilde{Z}_{\varepsilon}\right)=\emptyset, \forall k \geq 4$. 


\section{ESTRUCTURA LOCAL ALREDEDOR DE LOS PUNTOS DE TANGEN- CIA}

En la presente sección probaremos que el transformado estricto de un campo $Z \in \mathcal{D}_{v}^{3}$ tiene una estructura local bastante simple alrededor de los puntos de tangencia.

Definición 4.1 Sea $\beta$ una función que satisface las condiciones establecidas en (8). Decimos que $Z \in \mathcal{D}_{v}^{n}$ satisface la Condición $L$ si y sólo si para cada $2 \leq s \leq n$, el mapeo

$$
\Phi_{s}=\left(\beta, \tilde{Z} \beta, \ldots, \tilde{Z}^{s-1} \beta\right): \mathbb{C}^{n} \rightarrow \mathbb{C}^{s}
$$

tiene al $0 \in \mathbb{C}^{s}$ como un valor regular.

Observación. Si $Z \in \mathcal{D}_{v}^{n}$ la condición $\mathrm{L}$, entonces $\Gamma_{k}(\tilde{Z})=\emptyset, \forall k \geq n+1$ y los conjuntos $\Gamma_{1}(\tilde{Z}), \Gamma_{2}(\tilde{Z}), \ldots, \Gamma_{n}(\tilde{Z})$ son subvariedades algebraicas del divisor $D$.

Cuando $n=3$, existe una caracterización de la condición $\mathrm{L}$ en términos de las curvas $S$ and $S^{(1)}$.

Proposición 4.1 $Z \in \mathcal{D}_{v}^{n}$ satisface la condición L, si y sólo si $S$ no tiene puntos singulares y $S^{(1)}$ es transversal a $S$.

Demostración. La proposición es una consecuencia de los siguientes resultados:

1. $0 \in \mathbb{C}^{2}$ es un verdadero valor regular de $\Phi_{2}=(\beta, \tilde{Z} \beta)$ si y sólo si $S$ no tiene puntos singulares.

2. $0 \in \mathbb{C}^{3}$ es un verdadero valor regular de $\Phi_{3}=\left(\beta, \tilde{Z} \beta, \tilde{Z}^{2} \beta\right)$ si y sólo si $S^{(1)}$ en transversal a $S$.

De los Lemas 1 and 2, tenemos

$$
\begin{gathered}
\tilde{Z} \beta(y)=G_{0}(\hat{y})+y_{3} F_{1,1}(y) \\
\tilde{Z}^{2} \beta(y)=G_{1}(\hat{y})+(2-v) Z_{v+1}^{3}(\hat{y}) G_{0}(\hat{y})+y_{3} F_{2.2}(y)
\end{gathered}
$$

Observe que $\Phi_{2}^{-1}(0)=S$ and $\Phi_{3}^{-1}(0)=S \cap S^{(1)}$. 
Prueba de (i). De (23) se sigue que $0 \in \mathbb{C}^{2}$ es un valor regular de $\Phi_{2}$ si y sólo si $\nabla \beta(p)=(0,0,1)$ y $\nabla(\tilde{Z} \beta)(p)=\left(\frac{\partial(\tilde{Z} \beta)}{\partial y_{1}}(p), \frac{\partial(\tilde{Z} \beta)}{\partial y_{2}}(p), \frac{\partial(\tilde{Z} \beta)}{\partial y_{3}}(p)\right)$ son linealmente independientes $\forall p \in \Phi_{2}^{-1}(0)$ lo cual es equivalente a decir que $\left(\frac{\partial G_{0}}{\partial z_{1}}(\hat{y}), \frac{\partial G_{0}}{\partial z_{2}}(\hat{y})\right) \neq(0,0), \forall\left(y_{1}, y_{2}\right) \in S$ si y sólo si $S$ no tiene puntos singulares.

Prueba de (ii). De (24) tenemos que $0 \in \mathbb{C}^{s}$ es un valor regular de $\Phi_{3}$ si y sólo si

$$
\operatorname{det}\left(\begin{array}{ll}
\frac{\partial(\tilde{Z} \beta)}{\partial y_{1}}(p) & \frac{\partial(\tilde{Z} \beta)}{\partial y_{2}}(p) \\
\frac{\partial\left(\tilde{Z}^{2} \beta\right)}{\partial y_{1}}(p) & \frac{\partial\left(\tilde{Z}^{2} \beta\right)}{\partial y_{2}}(p)
\end{array}\right) \neq 0, \quad \forall p \in \Phi_{3}^{-1}(0)
$$

si y sólo si

$$
\operatorname{det}\left(\begin{array}{ll}
\frac{\partial G_{0}}{\partial z_{1}}(\hat{y}) & \frac{\partial G_{0}}{\partial z_{2}}(\hat{y}) \\
\frac{\partial G_{1}}{\partial z_{1}}(\hat{y}) & \frac{\partial G_{1}}{\partial z_{2}}(\hat{y})
\end{array}\right) \neq 0, \quad \forall\left(y_{1}, y_{2}\right) \in S \cap S^{(1)}
$$

si y sólo si $S^{(1)}$ es transversal a $S$.

Denotaremos por $\mathcal{D}_{v}^{n}(L)$ al conjunto de todos los campos vectoriales holomorfos $Z \in \mathcal{D}_{v}^{n}$ tales que $Z$ satisface la Condición L.

Teorema $4.2 \mathcal{D}_{v}^{3}(L)$ es abierto y denso en $\mathcal{D}_{v}^{3}$.

Demostración. Es claro que $\mathcal{D}_{v}^{3}(L)$ es un subconjunto abierto y denso en $\mathcal{D}_{v}^{3}$. La densidad es una consecuencia inmediata del Teorema 3.4 y la Proposición 4.1. En efecto, si $Z \in \mathcal{D}_{v}^{3}$ entonces existe $Z_{\varepsilon} \in \mathcal{D}_{v}^{3}$ suficientemente próximo a $Z$ tal que $S_{\varepsilon}$ es una curva algebraica no singular y $d s S_{\varepsilon}^{(1)}$ es transversal a $S_{\varepsilon}$, pero esto significa que $Z_{\varepsilon} \in \mathcal{D}_{v}^{3}(L)$. 
Con el fin de describir la estructura local alrededor de los puntos del divisor, necesitamos el siguiente resultado, el cual es una consecuencia directa del Teorema de Preparación de Weierstrass.

Proposición 4.3 Suponga que $Z \in \mathcal{D}_{v}^{n}$ satisface la Condición L. Si $p_{0} \in \Gamma_{k}(\tilde{Z})$, entonces podemos encontrar un cambio de coordenadas $t, x_{1}, \ldots, x_{n-1}$ definido en una vecindad $\tilde{U}$ de $p_{0}$ cual se anula en $p_{0}$ tal que $\left.\tilde{Z}\right|_{\tilde{U}}=\frac{\partial}{\partial t}$ y $D \cap \tilde{U}$ es dado por $\tilde{\gamma}^{-1}(0)$ donde

$$
\tilde{\gamma}(p)=t(p)^{k}+\sum_{j=1}^{k-1} x_{j}(p) t(p)^{j-1} .
$$

Cabe mencionar que en el caso real, la Proposición 4.3 fue probada independientemente por P. Percell [15] y J. Sotomayor [17] usando el Teorema de Preparación de Malgrange Mather.

Cuando $Z \in \mathcal{D}_{v}^{3}(L)$, del Teorema 4.2 y la Proposición 4.3, tenemos los tres casos siguientes:

1. Si $p_{0} \in \Gamma_{1}(\tilde{Z})$, entonces el divisor es dado localmente por

$$
D=\left\{\left(t, x_{1}, x_{2}\right): t=0\right\} .
$$

2- Si $p_{0} \in \Gamma_{2}(\tilde{Z})$, entonces $D=\left\{\left(t, x_{1}, x_{2}\right): t^{2}+x_{1}=0\right\}$.

3. Si $p_{0} \in \Gamma_{3}(\tilde{Z})$, entonces $D=\left\{\left(t, x_{1}, x_{2}\right): t^{3}+x_{1}+x_{2} t=0\right\}$.

Se sigue inmediatamente que si $Z_{1}, Z_{2} \in \mathcal{D}_{v}^{3}(L)$ y $p_{1} \in \Gamma_{k}\left(\tilde{Z}_{1}\right)$, $p_{2} \in \Gamma_{k}\left(\tilde{Z}_{2}\right)(1 \leq k \leq 3)$, entonces $Z_{1}$ y $\tilde{Z}_{2}$ son localmente topológicamente eequivalentes en $p_{1}$ and $p_{2}$.

Finalmente, introducimos la siguiente relación de equivalencia en $\mathcal{D}_{v}^{n}$.

Decimos que $Z_{1}, Z_{2} \in \mathcal{D}_{v}^{n}$ son Infinitesimalmente (topológicamente) equivalentes si y sólo si $\tilde{Z}_{1}$ y $\tilde{Z}_{2}$ son topológicamente equivalentes por un homeomorfismo que 
preserva el divisor. Observe que dos campos vectoriales infinitesimalmente (topológicamente) equivalentes $Z_{1}$ y $Z_{2}$ son localmente topológicamente equivalentes en $0 \in \mathbb{C}^{n}$.

Teorema 4.4 Cualquier par de elementos en $\mathcal{D}_{v}^{3}(L)$ son infinitesimalmente (topológicamente) equivalentes.

La prueba de Teorema 4.4 es completamente análoga al caso real (ver el Teorema 2 de [9]). De esta manera, hemos probado que cualquier par de elementos de $\mathcal{D}_{v}^{3}$ que satisface la condición $\mathrm{L}$ son topológicamente equivalentes y su estructura local es bastante simple.

\section{BIBLIOGRAFIA}

[1] R. Benazic, Caracterización de Singularidades dicríticas en foliaciones de dimensión uno, PESQUIMAT, Vol. I, Nº 1, (1998), pp. 73-81.

[2] R. Benazic, Sobre el Número de Milnor, PESQUIMAT, Vol. III, No 1, (2000), pp. 1-15.

[3] R. Benazic, A Resolution Theorem for Absolutely Isolated Singularities of Holomorphic Vector Fields, (1997).

[4] R. Benazic, Invarianza Topológica del Índice de Intersección, PESQUIMAT, Vol. IV, No 1, (2001), pp. 31-38.

[5] R. Benazic, Isolated Dicritical Singularities of a Holomorphic Vector Field, Tese IMPA, (1996).

[6] E. Brieskorn, H. Knórrer, Plane Algebraic Curves, Birkhauser-Verlag (1986).

[7] C. Camacho, F. Cano, P. Sad, Absolutely Isotated Singularities of Holomorphic Vector Fields, Invest. math. 98, (1989), pp. 351-369.

[8] F. Cano, Desingularization Strategies for 3-dimensional Vector Fields, Lecture Notes in Math. Vol. 1259. Berlin Heidelberg, New York; Springer (1987).

[9] M. I. Camacho, On the Local Structure of Real Vector Fields at a Dicritical Singularities.

[10] D. Cerveau, J. Mattei, Formes Intégrables Holomorphes Singularières, Asterisque, 97, (1982). 
[11] E. Chirka, Complex Analytic Sets, MIA, Kluwer Academic Publishers. Dordrecht, Boston, London (1989).

[12] P. Griffiths, J. Harris, Principles of Algebraic Geometric, Wiley - Intersciencie, New York, (1978).

[13] R. Hartshorne, Algebraic Geometric, New York, Springer Verlag (1977).

[14] J. Mattei, R. Moussu, Holonomie et Intégrales Premières, Ann Sci. Ecole. Norm. Sup. (4) 13 (1980) pp. 469-523.

[15] P. Percell, Structural Stability on Manifold with Boundary, Topology, Vol. 12, (1973).

[16] A. Seidenberg, Reduction of Singularities of the Differentiable Equation Ady $=B d x$, Amer. J. Math. 90 (1986) pp. 248-269.

[17] J. Sotomayor, Structural Stability in Manifolds with Boundary, Global Analysis and its applications, Vol. III, IAEA-SMR (1974). 